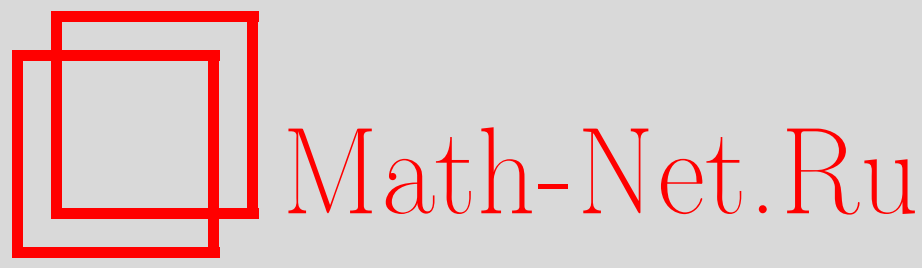

Л. В. Богданов, О классе многомерных интегрируемых иерархий и их редукциях, ТМФ, 2009, том 160, номep 1, 15-22

DOI: https://doi.org/10.4213/tmf6374

Использование Общероссийского математического портала Math-Net.Ru подразумевает, что вы прочитали и согласны с пользовательским соглашением http://www.mathnet.ru/rus/agreement

Параметры загрузки:

IP : 54.224.135.184

26 апреля 2023 г., 12:26:54

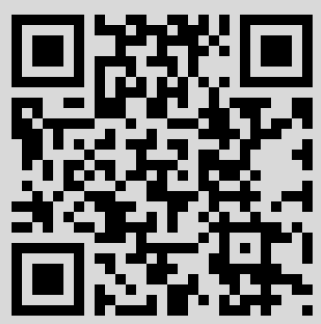




\title{
О КЛАССЕ МНОГОМЕРНЫХ ИНТЕГРИРУЕМЫХ ИЕРАРХИЙ И ИХ РЕДУКЦИЯХ
}

\begin{abstract}
Рассматривается класс многомерных интегрируемых иерархий, связанный с коммутативностью общих (нередуцированных) $(N+1)$-мерных векторных полей, содержащих производную по спектральной переменной. Эти иерархии определяются производящим уравнением, в том числе в форме Лакса-Сато. Для рассматриваемого класса иерархий приводится схема одевания, основанная на нелинейной векторной задаче Римана. В качестве характерных примеров рассматриваются иерархии, связанные с уравнением Манакова-Сантини и с системой Дунайского.
\end{abstract}

Ключевые слова: интегрируемая иерархия, бездисперсионное уравнение, "небесное уравнение", метод одевания.

\section{1. ВВЕДЕНИЕ}

Используя конструкцию, развитую в работах [1], [2], мы описываем структуру общей интегрируемой иерархии, связанной с коммутацией $(N+1)$-мерных векторных полей, которая определяет $(N+2)$-мерные интегрируемые уравнения. Мы рассматриваем несколько частных случаев общей иерархии и ее редукций; некоторые из них определяют известные интегрируемые модели. Простейший случай $(1+1)$-мерных векторных полей (одна пространственная переменная и одна спектральная переменная) соответствует $(2+1)$-мерной интегрируемой системе, введенной в работе [3] (см. также работы [4], [5]). Случай $(2+1)$-мерных векторных полей (две пространственные переменные и одна спектральная переменная) при редукции к сохраняющим объем векторным полям приводит к $(2+2)$-мерной интегрируемой модели Дунайского [6], описывающей антиавтодуальные уравнения Эйнштейна-Максвелла в сигнатуре $(2,2)$ (твисторная постановка).

\section{2. ОБЩАЯ $(N+2)$-МЕРНАЯ ИЕРАРХИЯ}

В настоящей работе мы избрали формальный путь введения иерархии, начинающийся с производящего уравнения, которое играет роль, подобную билинейному

${ }^{*}$ Институт теоретической физики им. Л. Д. Ландау РАН, Москва, Россия. E-mail: leonid@landau.ac.ru 
тождеству Хироты. Мы показываем, что производящее уравнение иерархии эквивалентно форме Лакса-Сато, которая дает нам линейные уравнения иерархии и нелинейные уравнения в частных производных, возникающие как условия их совместности. Линейные операторы имеют вид векторных полей, зависящих от спектральной переменной и включающих производную по спектральной переменной. Ввести иерархию другим способом, подобно тому, как это сделано в работах [4], [5], мы могли бы, начав с общей нелинейной векторной задачи Римана. Мы обсуждаем нелинейное векторное одевание Римана в конце нашей статьи.

Чтобы определить общую иерархию, мы рассматриваем $N+1$ формальных рядов, зависящих от $N$ бесконечных наборов дополнительных переменных (“времен”),

$$
\begin{aligned}
\Psi^{0} & =\lambda+\sum_{n=1}^{\infty} \Psi_{n}^{0}\left(\mathbf{t}^{1}, \ldots, \mathbf{t}^{N}\right) \lambda^{-n}, \\
\Psi^{k} & =\sum_{n=0}^{\infty} t_{n}^{k}\left(\Psi^{0}\right)^{n}+\sum_{n=1}^{\infty} \Psi_{n}^{k}\left(\mathbf{t}^{1}, \ldots, \mathbf{t}^{N}\right)\left(\Psi^{0}\right)^{-n},
\end{aligned}
$$

где $1 \leqslant k \leqslant N, \mathbf{t}^{k}=\left(t_{0}^{k}, \ldots, t_{n}^{k}, \ldots\right)$. Мы вводим обозначения $\partial_{n}^{k}$ для $\partial / \partial t_{n}^{k}$ и $\Psi$ для вектора (столбца) с компонентами $\Psi^{0}, \ldots, \Psi^{N}$, а также проекторы

$$
\left(\sum_{-\infty}^{\infty} u_{n} \lambda^{n}\right)_{+}=\sum_{n=0}^{\infty} u_{n} \lambda^{n}, \quad\left(\sum_{-\infty}^{\infty} u_{n} \lambda^{n}\right)_{-}=\sum_{-\infty}^{n=-1} u_{n} \lambda^{n} .
$$

Общая иерархия определяется производящим соотношением

$$
\left(J_{0}^{-1} d \Psi^{0} \wedge d \Psi^{1} \wedge \cdots \wedge d \Psi^{N}\right)_{-}=0
$$

где дифференциал включает как времена, так и спектральную переменную,

$$
d f=\sum_{k=1}^{N} \sum_{n=0}^{\infty} \partial_{n}^{k} f d t_{n}^{k}+\partial_{\lambda} f d \lambda
$$

в формуле (3) $J_{0}$ - детерминант матрицы Якоби $J$,

$$
J_{i j}=\partial_{i} \Psi^{j}, \quad 0 \leqslant i, j \leqslant N
$$

где

$$
\partial_{0}=\frac{\partial}{\partial \lambda}, \quad \partial_{k}=\frac{\partial}{\partial x^{k}}, \quad x^{k}=t_{0}^{k}, \quad 1 \leqslant k \leqslant N .
$$

Уравнение (3) является производящим соотношением для формы Лакса-Сато иерархии.

ПрЕДЛОЖЕНИЕ. Соотношение (3) эквивалентно бесконечному набору уравнений

$$
\partial_{n}^{k} \boldsymbol{\Psi}=\sum_{i=0}^{N}\left(J_{k i}^{-1}\left(\Psi^{0}\right)^{n}\right)_{+} \partial_{i} \boldsymbol{\Psi}, \quad 0 \leqslant n \leqslant \infty, \quad 1 \leqslant k \leqslant N .
$$


Доказательство этого предложения полностью аналогично доказательству для иерархии Дунайского, приведенному в работе [2]. Доказательство того, что из (3) следует иерархия (4), основано на следующем утверждении.

ЛЕмма 1. Если дано тождество (3), то для произволъного оператора первого порядка

$$
\widehat{U}=\sum_{k=1}^{N} \sum_{i} u_{i}^{k}(\lambda, \mathbf{t}) \partial_{i}^{k}+u^{0}\left(\lambda, \mathbf{t}^{1}, \mathbf{t}^{2}\right) \partial_{\lambda}
$$

c “плюсовыми” коэфбициентами $\left(\left(u_{i}^{k}\right)_{-}=u_{-}^{0}=0\right)$ условие $(\widehat{U} \boldsymbol{\Psi})_{+}=0$ (где для $\Psi^{k}, k \neq 0$, производные рядов (2) берутся при фиксированном $\Psi^{0}$ ) влечет за собой равенство $\widehat{U} \boldsymbol{\Psi}=0$.

ДокАЗАТЕЛЬСтво. В полной аналогии с работами [4], [5] из производящего соотношения (3) следует линейная система $\left(J^{\mathrm{T}}\right)^{-1} \widehat{U} \boldsymbol{\Psi}=0$ на $\widehat{U} \boldsymbol{\Psi}$. Принимая во внимание то, что $\operatorname{det} J \neq 0\left(\operatorname{det} J=1+\sum_{p=1}^{N} \partial_{p} \Psi_{1}^{p} / \lambda+\cdots\right)$, мы получаем, что эта система имеет единственное решение $\widehat{U} \boldsymbol{\Psi}=0$.

Следствие $(4) \Rightarrow(3)$ непосредственно вытекает из следующего утверждения.

Лемма 2. Имеет место соотношение

$$
\operatorname{det}\left(\frac{\partial}{\partial \tau_{i}} \Psi_{j}\right)=J_{0} \operatorname{det}\left(V_{\tau_{i}+}^{j}\right),
$$

где $\tau_{i}, 0 \leqslant i \leqslant N$, представляют собой произвольный набор времен иерархии, a $V_{\tau_{i}+}^{j}-$ коэфбициенты соответствующих векторных полей, заданных правой частъю уравнений (4),

$$
\partial_{\tau_{i}} \boldsymbol{\Psi}=\sum_{j=0}^{N} V_{\tau_{i}+}^{j} \partial_{j} \Psi
$$

Аналогично работе [2] можно также доказать, что потоки Лакса-Сато (4) общей иерархии совместны.

Утверждение леммы 1 является основным инструментом конструирования линейных уравнений иерархии в обычном духе метода одевания. Сначала мы выбираем базис, по которому можно разложить произвольную сингулярную ("плюсовую") часть линейного оператора, действующего на $\boldsymbol{\Psi}$, и который в нашем случае образован набором функций $\lambda^{n} \partial_{k} \boldsymbol{\Psi}, \lambda^{n} \partial_{\lambda} \boldsymbol{\Psi}, 0 \leqslant n<\infty, 0<k \leqslant N$. Затем берем производную $\boldsymbol{\Psi}$ по произвольному "высшему" времени иерархии и, разлагая ее по базису и используя лемму 1 , получаем некоторое линейное уравнение иерархии. Уравнения (4) имеют в точности такой вид, и единственное, что нам нужно для доказательства их справедливости - это проверить отсутствие сингулярности (“плюсового" члена). Используя разложение по базису, легко получить первые линейные уравнения иерархии непосредственно (другим способом их получения является использование общих формул (4)):

$$
\partial_{1}^{k} \boldsymbol{\Psi}=\left(\lambda \partial_{k}-\sum_{p=1}^{N}\left(\partial_{k} u_{p}\right) \partial_{p}-\left(\partial_{k} u_{0}\right) \partial_{\lambda}\right) \boldsymbol{\Psi}, \quad 0<k \leqslant N
$$


где $u_{k}=\Psi_{1}^{k}, 0 \leqslant k \leqslant N$. Условие совместности любой пары линейных уравнений (6) (например, с $\partial_{1}^{k}$ и $\partial_{1}^{q}, k \neq q$ ) дает замкнутую нелинейную $(N+2)$-мерную систему дифференциальных уравнений в частных производных для множества функций $u_{k}$, $u_{0}$, которая может быть записана в виде

$$
\begin{gathered}
\partial_{1}^{k} \partial_{q} \hat{u}-\partial_{1}^{q} \partial_{k} \hat{u}+\left[\partial_{k} \hat{u}, \partial_{q} \hat{u}\right]=\left(\partial_{k} u_{0}\right) \partial_{q}-\left(\partial_{q} u_{0}\right) \partial_{k} \\
\partial_{1}^{k} \partial_{q} u_{0}-\partial_{1}^{q} \partial_{k} u_{0}+\left(\partial_{k} \hat{u}\right) \partial_{q} u_{0}-\left(\partial_{q} \hat{u}\right) \partial_{k} u_{0}=0
\end{gathered}
$$

где $\hat{u}$ - векторное поле, $\hat{u}=\sum_{p=1}^{N} u_{k} \partial_{k}$. В случае $u_{0}=0$ мы имеем только первое уравнение без правой части. Этот случай соответствует общим векторным полям, не содержащим производную по спектральной переменной, и при гамильтоновой редукции представляет собой гиперкэлеровы иерархии [7], [8]. Структура общей системы (7) в основе такая же, как структура предложенного Дунайским обобщения второго "небесного" уравнения (heavenly equation) [6].

\section{3. ИЕРАРХИЯ МАНАКОВА-САНТИНИ}

Простейший случай общей иерархии (4) при $N=1$ связан с системой, недавно предложенной в статье [3],

$$
\begin{aligned}
& u_{x t}=u_{y y}+\left(u u_{x}\right)_{x}+v_{x} u_{x y}-u_{x x} v_{y}, \\
& v_{x t}=v_{y y}+u v_{x x}+v_{x} v_{x y}-v_{x x} v_{y},
\end{aligned}
$$

пара Лакса для которой есть

$$
\begin{aligned}
& \partial_{y} \boldsymbol{\Psi}=\left(\left(\lambda-v_{x}\right) \partial_{x}-u_{x} \partial_{\lambda}\right) \boldsymbol{\Psi}, \\
& \partial_{t} \boldsymbol{\Psi}=\left(\left(\lambda^{2}-v_{x} \lambda+u-v_{y}\right) \partial_{x}-\left(u_{x} \lambda+u_{y}\right) \partial_{\lambda}\right) \boldsymbol{\Psi} .
\end{aligned}
$$

Эта система была найдена при изучении задачи Коши для бездисперсионного уравнения КП (уравнения Хохлова-Заболоцкой)

$$
u_{x t}=u_{y y}+\left(u u_{x}\right)_{x} .
$$

Хорошо известно, что пара Лакса для этого уравнения может быть записана в терминах гамильтоновых векторных полей, и динамика связана с группой $\operatorname{Sdiff}(2)$ :

$$
\begin{array}{rlrl}
\partial_{y} \boldsymbol{\Psi} & =\left(\left(\partial_{\lambda} H^{y}\right) \partial_{x}-\left(\partial_{x} H^{y}\right) \partial_{\lambda}\right) \boldsymbol{\Psi}, & H^{y}=\frac{\lambda^{2}}{2}+u, \\
\partial_{t} \boldsymbol{\Psi}=\left(\left(\partial_{\lambda} H^{t}\right) \partial_{x}-\left(\partial_{x} H^{t}\right) \partial_{\lambda}\right) \boldsymbol{\Psi}, & H^{t}=\frac{\lambda^{3}}{3}+\lambda u+w, \quad w_{x}=u_{y} .
\end{array}
$$

Однако в терминах нелинейного одевания Римана-Гильберта, используемого в работе [3] (см. также статьи [4], [5]), гамильтоновость векторных полей является просто редукцией к данным одеваниям, и возможно также рассмотреть случай общих (нередуцированных) векторных полей. Это наблюдение привело к открытию системы (8). При $v=0$ эта система редуцируется к бездисперсионному уравнению КП (9). Соответственно, редукция $u=0$ дает уравнение [9] (см. также [10]-[12])

$$
v_{x t}=v_{y y}+v_{x} v_{x y}-v_{x x} v_{y} .
$$


Мы покажем, что иерархия, связанная с системой (8), представляет собой частный случай общей иерархии (4) при $N=1$. В этом случае мы имеем два ряда

$$
\begin{aligned}
& \Psi^{0}=\lambda+\sum_{n=1}^{\infty} \Psi_{n}^{0}(\mathbf{t}) \lambda^{-n} \\
& \Psi^{1}=\sum_{n=0}^{\infty} t_{n}\left(\Psi^{0}\right)^{n}+\sum_{n=1}^{\infty} \Psi_{n}^{1}(\mathbf{t})\left(\Psi^{0}\right)^{-n}
\end{aligned}
$$

и производящее соотношение

$$
\left(J_{0}^{-1} d \Psi^{0} \wedge d \Psi^{1}\right)_{-}=0 .
$$

Уравнения Лакса-Сато для этой иерархии имеют вид

$$
\partial_{n} \boldsymbol{\Psi}=\sum_{i=0,1}\left(J_{1 i}^{-1}\left(\Psi^{0}\right)^{n}\right)_{+} \partial_{i} \boldsymbol{\Psi}, \quad 0 \leqslant n \leqslant \infty
$$

где

$$
J=\left(\begin{array}{ll}
\Psi_{\lambda}^{0} & \Psi_{\lambda}^{1} \\
\Psi_{x}^{0} & \Psi_{x}^{1}
\end{array}\right),
$$

и

$$
\begin{aligned}
J_{10}^{-1} & =-J_{0}^{-1} \Psi_{x}^{0}, \quad J_{11}^{-1}=J_{0}^{-1} \Psi_{\lambda}^{0}, \\
J_{0} & =\operatorname{det} J=1+\partial_{x} \Psi_{1}^{1} \lambda^{-1}+\left(\partial_{x} \Psi_{2}^{1}-\Psi_{1}^{0}\right) \lambda^{-2}+\cdots .
\end{aligned}
$$

В более явной форме уравнения Лакса-Сато записываются как

$$
\partial_{n} \boldsymbol{\Psi}=\left(J_{0}^{-1} \Psi_{\lambda}^{0}\left(\Psi^{0}\right)^{n}\right)_{+} \partial_{x} \boldsymbol{\Psi}-\left(J_{0}^{-1} \Psi_{x}^{0}\left(\Psi^{0}\right)^{n}\right)_{+} \partial_{\lambda} \boldsymbol{\Psi}
$$

Уравнения Лакса-Сато для первых двух потоков имеют вид

$$
\begin{aligned}
& \partial_{y} \boldsymbol{\Psi}=\left(\left(\lambda-v_{x}\right) \partial_{x}-u_{x} \partial_{\lambda}\right) \boldsymbol{\Psi} \\
& \partial_{t} \boldsymbol{\Psi}=\left(\left(\lambda^{2}-v_{x} \lambda+u-v_{y}\right) \partial_{x}-\left(u_{x} \lambda+u_{y}\right) \partial_{\lambda}\right) \boldsymbol{\Psi}
\end{aligned}
$$

где $u=\Psi_{1}^{0}, v=\Psi_{1}^{1}, x=t_{0}, y=t_{1}, t=t_{2}$. Условия совместности этих уравнений представляют собой систему (8). Таким образом, общая иерархия (4) при $N=1$, заданная уравнениями (14), определяет иерархию коммутирующих потоков для системы (8), так что ее можно назвать иерархией Манакова-Сантини.

Для того чтобы редуцировать иерархию Манакова-Сантини (14) к бездисперсионной иерархии КП, необходимо рассмотреть редукцию $J_{0}=1$ (условие сохранения площади или гамильтоновости), тогда из уравнений Лакса-Сато (14) легко получаются уравнения Лакса-Сато бездисперсионной иерархии КП. Редукция $\Psi^{0}=\lambda$ приводит к иерархии, связанной с уравнением (10), которая рассматривалась в работах [11], [12]. 


\section{4. ИЕРАРХИЯ УРАВНЕНИЯ ДУНАЙСКОГО}

Данная иерархия связана с предложенной Дунайским [6] интегрируемой моделью, обобщающей знаменитое второе "небесное" уравнение Плебанского. Она соответствует общей иерархии при $N=2$ с редукцией, сохраняющей объем $\left(J_{0}=1\right)$. Чтобы определить эту иерархию, введем три ряда

$$
\begin{aligned}
& \Psi^{0}=\lambda+\sum_{n=1}^{\infty} \Psi_{n}^{0}\left(\mathbf{t}^{1}, \mathbf{t}^{2}\right) \lambda^{-n}, \\
& \Psi^{1}=\sum_{n=0}^{\infty} t_{n}^{1}\left(\Psi^{0}\right)^{n}+\sum_{n=1}^{\infty} \Psi_{n}^{1}\left(\mathbf{t}^{1}, \mathbf{t}^{2}\right)\left(\Psi^{0}\right)^{-n}, \\
& \Psi^{2}=\sum_{n=0}^{\infty} t_{n}^{2}\left(\Psi^{0}\right)^{n}+\sum_{n=1}^{\infty} \Psi_{n}^{2}\left(\mathbf{t}^{1}, \mathbf{t}^{2}\right)\left(\Psi^{0}\right)^{-n} .
\end{aligned}
$$

Производящее уравнение иерархии Дунайского имеет вид

$$
\left(d \Psi^{0} \wedge d \Psi^{1} \wedge d \Psi^{2}\right)_{-}=0
$$

(мы учли редукцию $J_{0}=1$ ). Уравнения Лакса-Сато иерархии записываются как

$$
\begin{aligned}
\partial_{n}^{1} \boldsymbol{\Psi}= & \left(\left(\Psi^{0}\right)^{n}\left|\begin{array}{ll}
\Psi_{\lambda}^{0} & \Psi_{\lambda}^{2} \\
\Psi_{y}^{0} & \Psi_{y}^{2}
\end{array}\right|\right)_{+} \partial_{x} \boldsymbol{\Psi}-\left(\left(\Psi^{0}\right)^{n}\left|\begin{array}{cc}
\Psi_{\lambda}^{0} & \Psi_{\lambda}^{2} \\
\Psi_{x}^{0} & \Psi_{x}^{2}
\end{array}\right|\right)_{+} \partial_{y} \boldsymbol{\Psi}- \\
& -\left(\left(\Psi^{0}\right)^{n}\left|\begin{array}{ll}
\Psi_{x}^{0} & \Psi_{x}^{2} \\
\Psi_{y}^{0} & \Psi_{y}^{2}
\end{array}\right|\right)_{+} \partial_{\lambda} \boldsymbol{\Psi} \\
\partial_{n}^{2} \boldsymbol{\Psi}= & -\left(\left(\Psi^{0}\right)^{n}\left|\begin{array}{ll}
\Psi_{\lambda}^{0} & \Psi_{\lambda}^{1} \\
\Psi_{y}^{0} & \Psi_{y}^{1}
\end{array}\right|\right)_{+} \partial_{x} \boldsymbol{\Psi}+\left(\left(\Psi^{0}\right)^{n}\left|\begin{array}{cc}
\Psi_{\lambda}^{0} & \Psi_{\lambda}^{1} \\
\Psi_{x}^{0} & \Psi_{x}^{1}
\end{array}\right|\right)_{+} \partial_{y} \boldsymbol{\Psi}+ \\
& +\left(\left(\Psi^{0}\right)^{n}\left|\begin{array}{ll}
\Psi_{x}^{0} & \Psi_{x}^{1} \\
\Psi_{y}^{0} & \Psi_{y}^{1}
\end{array}\right|\right)_{+} \partial_{\lambda} \boldsymbol{\Psi}
\end{aligned}
$$

(с дополнительным условием $J_{0}=1$ ). Легко проверить, что при $\Psi^{0}=\lambda$ иерархия уравнения Дунайского сводится к иерархии "небесного" уравнения [7], [8], в то время как при $\Psi^{2}=y$ она редуцируется к бездисперсионной иерархии КП. Первые два потока иерархии (16), (17) имеют вид

$$
\begin{aligned}
\partial_{1}^{1} \boldsymbol{\Psi} & =\left(u_{y} \partial_{x}-u_{x} \partial_{y}+\lambda \partial_{x}-f_{x} \partial_{\lambda}\right) \boldsymbol{\Psi}, \\
\partial_{1}^{2} \boldsymbol{\Psi} & =\left(v_{x} \partial_{y}-v_{y} \partial_{x}+\lambda \partial_{y}-f_{y} \partial_{\lambda}\right) \boldsymbol{\Psi} ;
\end{aligned}
$$

здесь $u=\Psi_{1}^{2}, v=\Psi_{1}^{1}, f=\Psi_{1}^{0}$. Из условия $\operatorname{det} J=1$ следует, что $u_{y}+v_{x}=0$, и мы можем ввести потенциал $\Theta$ такой, что $v=\Theta_{y}, u=-\Theta_{x}$. При отождествлении $z=-t_{1}^{1}, w=t_{1}^{2}$ мы получаем пару Лакса для системы Дунайского:

$$
\begin{aligned}
\Theta_{w x}+\Theta_{z y}+\Theta_{x x} \Theta_{y y}-\Theta_{x y}^{2} & =f, \\
f_{x w}+f_{y z}+\Theta_{y y} f_{x x}+\Theta_{x x} f_{y y}-2 \Theta_{x y} f_{x y} & =0 .
\end{aligned}
$$


Вторые потоки могут быть записаны как

$$
\begin{aligned}
& \partial_{2}^{1} \boldsymbol{\Psi}=\left(\lambda \partial_{1}^{1}+f \partial_{x}-\left(\partial_{1}^{1} f\right) \partial_{\lambda}\right) \boldsymbol{\Psi} \\
& \partial_{2}^{2} \boldsymbol{\Psi}=\left(\lambda \partial_{1}^{2}+f \partial_{y}-\left(\partial_{1}^{2} f\right) \partial_{\lambda}\right) \boldsymbol{\Psi} .
\end{aligned}
$$

Чтобы выписать векторные поля в более явном виде, необходимо использовать первые потоки (17), (19). Условие коммутативности любой пары потоков задает уравнение на $\Theta, f$ (с различным набором времен).

\section{5. СХЕМА ОДЕВАНИЯ}

Рассмотрим нелинейную векторную задачу Римана вида

$$
\boldsymbol{\Psi}_{+}=\mathbf{F}\left(\boldsymbol{\Psi}_{-}\right)
$$

где $\boldsymbol{\Psi}_{+}, \boldsymbol{\Psi}_{-}$обозначают граничные значения $(N+1)$-компонентной вектор-функции на сторонах некоторой ориентированной кривой $\gamma$ в комплексной плоскости переменной $\lambda$. Задача состоит в том, чтобы найти функцию, аналитическую вне кривой, с некоторым фиксированным поведением на бесконечности (нормировкой), которая удовлетворяет уравнению (20). Задача такого типа рассматривалась Такасаки [7], [8], [13], который подчеркивал ее связь с конструкцией нелинейного гравитона Пенроуза.

Задача (20) связана с классом интегрируемых уравнений, которые могут быть представлены как условия коммутативности векторных полей, содержащих производную по спектральной переменной. Мы приводим набросок схемы одевания, соответствующей общей $(N+2)$-мерной интегрируемой иерархии (3), (4). Сначала в качестве $\gamma$ берем единичную окружность. Чтобы получить решения общей иерархии с помощью задачи (20), необходимо найти решение этой задачи с особенностями при $\lambda=\infty$, определяемыми рядами (1), (2) (для простоты мы предполагаем, что только конечное число времен не равно нулю). Если для этого решения детерминант $J_{0}$ не равен нулю, то мы приходим к заключению, что форма

$$
\Omega=J_{0}^{-1} d \Psi^{0} \wedge d \Psi^{1} \wedge \cdots \wedge d \Psi^{N}
$$

аналитична в комплексной плоскости и, таким образом, удовлетворяет производящему тождеству (3). Тогда решение задачи Римана (20) удовлетворяет уравнениям Лакса-Сато (4) и дает решение общей $(N+2)$-мерной иерархии.

Вследствие того что особенности решения задачи Римана задаются рядами (1), (2) с использованием самого решения, решение иерархии обычно определяется в терминах неявных функций.

Благодарности. Автор частично поддержан РФФИ (гранты № 07-01-00446_a, 06-01-92053-КЭ_а и 08-01-90104-Мол_а). 


\section{Список литературы}

[1] L.V. Bogdanov, V.S. Dryuma, S. V. Manakov, On the dressing method for Dunajski anti-self-duality equation, arXiv: nlin/0612046.

[2] L. V. Bogdanov, V. S. Dryuma, S. V. Manakov, J. Phys. A, 40:48 (2007), 14383-14393.

[3] S. V. Manakov, P. M. Santini, Писъма в ЖКЭТФ, 83:10 (2006), 534-538.

[4] С. В. Манаков, П. М. Сантини, ТМФ, 152:1 (2007), 147-156.

[5] S. V. Manakov, P. M. Santini, J. Phys. A, 41:5 (2008), 055204.

[6] M. Dunajski, Proc. R. Soc. Lond. Ser. A, 458:2021 (2002), 1205-1222.

[7] K. Takasaki, J. Math. Phys., 30:7 (1989), 1515-1521.

[8] K. Takasaki, J. Math. Phys., 31:8 (1990), 1877-1888.

[9] M. V. Pavlov, J. Math. Phys., 44:9 (2003), 4134-4156.

[10] M. Dunajski, J. Geom. Phys., 51:1 (2004), 126-137.

[11] L. Martínez Alonso, A. B. Shabat, Phys. Lett. A, 300:1 (2002), 58-64.

[12] Л. Мартинес Алонсо, А. Б. Шабат, ТМФ, 140:2 (2004), 216-229.

[13] K. Takasaki, Phys. Lett. B, 285:3 (1992), 187-190. 